\title{
Hart am Limit
}

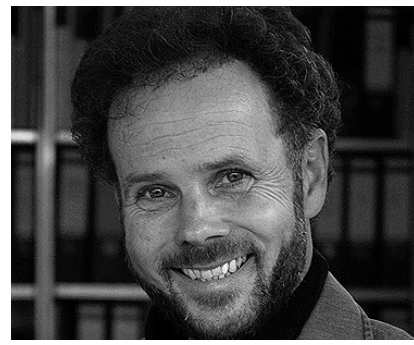

VON THOMAS MÄULE Dr. Thomas Mäule, Theologe, Sportwissenschaftler, Gerontologe und Sozialmanager, ist Referent für Theologie und Ethik bei der Evangelischen Heimstiftung $\mathrm{GmbH}$ in Stuttgart. Die Organisation ist mit 7.400 Plätzen und 6.400 hauptberuflichen Mitarbeiterinnen und Mitarbeitern der größte Anbieter von Altenhilfedienstleistungen in Baden-Württemberg.

E-Mail t.maeule@ev-heimstiftung.de

\section{An Führungskräfte werden hohe Erwartungen gestellt. Personelle Engpässe und Kostendruck fordern, eigene Grenzen weit auszudehnen. Wie aber gehen sie mit Stress und Belastung im Berufsalltag um? Die Evangelische Heimstiftung hat Hausdirektionen befragt und daraus Überlegungen zur Burn-out-Prävention abgeleitet.}

Wo Hausdirektionen mit hohem Engagement auf hohem Belastungsniveau arbeiten, ist die Gefahr des Ausbrennens groß. Was hält sie gesund? Gesundheitsförderndes Führen fängt bei der eigenen Person an. Nur gesunde Führungskräfte können ihrer Führungsaufgabe und Vorbildfunktion gerecht werden. Geht es den Führungskräften nicht gut, kann dies Organisationsstrukturen, betriebliche Rahmenbedingungen und das persönliche Miteinander tangieren. In einer Stellenanzeige heißt es:

»Als Hausdirektor/in übernehmen Sie die Aufgaben der Heimleitung und der verantwortlichen Pflegefachkraft. Aus diesem Grund benötigen Sie die Qualifikation zur Heimleitung nach $\mathbb{} 2$ HeimPersV und zur verantwortlichen Pflegefachkraft nach $\mathbb{S} 80$ SGB XI. Sie haben mehrjährige einschlägige Berufs- und Führungserfahrung. Sie identifizieren sich mit den Zielen und den Handlungsgrundsätzen des Trägers. Als Mitglied einer christlichen Kirche setzen Sie den diakonischen Auftrag in Ihrer täglichen Arbeit um. Wesentliche Aspekte Ihrer Tätigkeit sind die tägliche Kommunikation mit den Bewohnern und deren Angehörigen, die Koordination und Führung innerhalb der Einrichtung und die Repräsentation des Pflegezentrums gegenüber der Öffentlichkeit. Bei der wirtschaftlichen Steuerung arbeiten Sie eng mit dem Regionaldirektor der Region zusammen. «
Vor diesem Hintergrund hat die Geschäftsführung der Evangelischen Heimstiftung $\mathrm{GmbH}$ eine Repräsentativbefragung unter allen 66 Hausdirektionen beschlossen. Die Befragung soll Aufschluss geben, wie es Einrichtungsleitenden gelingt, auf Dauer gut für sich selbst zu sorgen und unter hohem Stressniveau nicht "auszubrennen «. Aber auch: welche konkreten Wünsche die Führungskräfte an den Träger zur Gesunderhaltung haben.

\section{Die Studie}

Die Befragung fand vom 28. Januar bis 22. Februar 2011 statt. Der Fragebogen enthielt offene, halboffene und geschlossene Fragen. Die Rückmeldungen wurden absolut vertraulich behandelt und ließen keinerlei Rückschlüsse auf die Person zu. Zum Abschluss der Erhebung lagen 41 Fragebögen vor. Eine Rücklaufquote von 62 Prozent entspricht einem außerordentlich hohen Respons. Die Auswertung erfolgte in Form einfacher, beschreibender Statistik. Die Datenlage erlaubt es, weitergehende Überlegungen darüber anzustellen, was erforderlich ist, dass Hausdirektionen verantwortungsvoll ihren Beruf ausüben und dennoch nicht ausbrennen. (Besonderer Dank gilt Herrn Stefan Bratsch, Student der Wirtschaftswissenschaft, für die Projektmitarbeit, die Auswertung und Darstellung der Ergebnisse.) 


\section{Einschätzung des Arbeitsumfeldes}

$\mathrm{Zu}$ Beginn des Fragebogens geht es um eine allgemeine Einschätzung des Arbeitsumfeldes:

n »Habe ich alles, was ich brauche, um die Aufgaben, vor die mich meine berufliche Tätigkeit stellt, zu bewältigen? « (»Handhabbar «)

- "Sind die Anforderungen, die im Rahmen meiner beruflichen Tätigkeit an mich gestellt werden, strukturiert, klar und eindeutig? « (»Übersichtlich «)

- »Sind die Aufgaben, die ich im Rahmen meiner beruflichen Tätigkeit erledigen muss, sehr wichtig? « (» Wich$\operatorname{tig} \ll)$

Von 71 Prozent der Hausdirektionen wird das Arbeitsumfeld als gut zu bewältigen empfunden. Defizite sehen die Befragten insbesondere bei der "Übersichtlichkeit " (39\%). Die Sinnhaftigkeit (»Wichtigkeit «) der Tätigkeit ist für die Hausdirektionen generell hoch (98\%).

Der Bewertung des allgemeinen Arbeitsumfeldes liegt eine Skala von 1 (»überhaupt nicht «) bis 5 (»vollkommen«) zugrunde. Basis der Auswertung
- »Was empfinden Sie als negativ, hinderlich oder demotivierend? «

- »Was nehmen Sie als positiv, hilfreich oder motivierend wahr? «

Als klare Stressoren, denen sich Hausdirektionen im beruflichen Alltag ausgesetzt sehen, lassen sich identifizieren:

- die vielen verschiedenen, unvorhersehbaren Aufgaben

- die zu langen, mit Überstunden einhergehenden Arbeitszeiten, ständige Erreichbarkeit und Entgrenzung von Arbeit und Freizeit

- ein Führungsstil, der von überzogenen Leistungserwartungen und wirtschaftlichem Druck, fehlender Anerkennung, unklarer Rollen- und Aufgabenverteilung und Konflikten geprägt ist

- die Vielzahl von Regelungen und Vorgaben; unklare Informations- und Kommunikationsstrukturen

Unterstützende Ressourcen sind insbesondere:

- Austausch und Unterstützung von Kolleginnen und Kollegen, insbesondere in derselben Region

\section{"Faire Rückmeldungen durch Vorgesetzte sind zentrale Momente eines gesunderhal- tenden Organisationsklimas"}

ist der Grad der Zustimmung zu den Aussagen. Die Fragestellungen lehnen sich an die Konzeption des »Kohärenzgefühls « an. Das Kohärenzgefühl (»sense of coherence ") ist Kernstück von Antonovskys Salutogenetischem Modell der Gesundheit. Kohärenz bedeutet Zusammenhang, Stimmigkeit. Je ausgeprägter das Kohärenzgefühl einer Person, desto gesünder ist sie.

\section{Berufliche Belastungssituation}

Um Belastungen und hilfreiche Ressourcen im Berufsalltag geht es im zweiten Fragenkomplex. Das Profil der Belastungssituation von Hausdirektionen ergibt sich aus der Analyse der Antworten auf die Fragen:
- ein gutes Arbeitsklima und ein Team, in dem Austausch und Offenheit möglich sind

- Gestaltungsfreiraum

- ein Führungsstil, der von Interesse und Wertschätzung, Rückmeldung und Rückendeckung geprägt ist

- Entlastung durch Fachabteilungen und Mitarbeitende der Hauptverwaltung

- Angebote zur Fort- und Weiterbildung sowie zur Begleitung

\section{Umgang mit Belastungen}

Um Gesunderhaltungsstrategien geht es im dritten Fragenkomplex:

घ »Welche Handlungsstrategien haben Sie bei Stress und Belastung? Was sind
Ihre persönlichen Potentiale/Stärken/ Kraftquellen zur Erhaltung von Gesundheit und Zufriedenheit? Wie halten Sie sich fit? «

Die Antworten sind zu Kategorien zusammengefasst und dem privaten sowie beruflichen Bereich zugeordnet.

Privater Bereich: 93 Prozent der Befragten geben an, sich im privaten Bereich gesund zu erhalten. Folgende Kraftquellen lassen sich ausmachen:

- Sport, Bewegung, Hobbys, Aktivitäten

- Familie, Partner, Freunde

- Spiritualität, Glaube, Gebet, Meditation

- Ruhe, Stille, Natur.

Beruflicher Bereich: Strategien zum Umgang mit Belastungen im beruflichen Bereich geben 68 Prozent der Hausdirektionen an. Wichtige Momente der Gesunderhaltung sind

- Gelassenheit und positive Grundhaltung

- kollegialer Austausch

- aktives Angehen von Problemen, Grenzen setzen

- Selbstreflexion und Selbstorganisation

- Hilfe von anderen annehmen

\section{Förderung der Gesundheit und Arbeitszufriedenheit}

Im vierten Fragenkomplex geht es um wünschenswerte Entlastung im Berufsalltag und konkretes Unterstützungspotenzial:

• »Wie können in Ihrem beruflichen Alltag Belastungen verringert werden? Was wären entlastende Lösungen? Was würde Sie konkret (mehr) unterstützen?«

Wünsche nach mehr Unterstützung und zur Gesunderhaltung richten sich auf:

- die Schärfung des Aufgabenprofils

- die Arbeitszeitgestaltung

- weniger Leistungsdruck

- einen von mehr Anerkennung gekennzeichneten Führungsstil

- intensiveren Austausch im Kreis der Kolleginnen und Kollegen

- bessere Unterstützung durch die Hauptverwaltung 
- den Abbau von Bürokratie

- die Möglichkeit zu mehr Reflexion über das eigene Tun durch Supervision und Coaching.

\section{Was wer tun kann: der Einzelne}

Gefordert für eine wirksame Burn-outPrävention sind insbesondere die Führungskraft selbst, sein Arbeitsumfeld und der Anstellungsträger.

Zunächst sind die Hausdirektionen selbst gefordert. Die vorliegenden Ergebnisse zeigen: Um Stresssituationen zu bewältigen, sind persönliche Einstellungen und Überzeugungen gefragt. Bei schwierigen Situationen sind Handlungsmuster vonnöten, um emotional stabil zu sein und gesund zu bleiben.

Antworten zu persönlichen Strategien verweisen auf einen ausgewogenen Lebensstil, Humor und die Fähigkeit, Hilfe zu erbitten, abgeben und loszulassen zu können. Viele profitieren von ihrer religiösen Verbundenheit. Zur Selbstsorge gehören gesunde Lebensweise und Entspannungstraining. Zur ausgeglichenen »Life-Work-Balance « führt ein Gleichgewicht aus Familie und Freundeskreis, Sport und Bewegung, Kultur und Engagement, Ruhe und Aktivität. Zur Selbstreflexion die Grundfragen: »Was treibt mich an? Wofür tue ich das wirklich? Wofür habe ich Zeit, wofür nicht? Wo liegen Prioritäten? «

Dafür muss man sich - auch dies eine Rückmeldung aus der Befragung - keinesfalls dem Leistungsgedanken entziehen. Die Wertschätzung der eigenen Gesundheit zeigt sich darin, dass körperliche Warnsignale nicht ignoriert, dass notwendige Auszeiten nicht vernachlässigt werden.

\section{Was wer tun kann: das Arbeitsumfeld}

Ansatzpunkte für die einzelne Hausdirektion im Kontext der Einrichtung beziehen sich auf:
- Arbeitsgestaltung: Eine wichtige Erkenntnis aus der Befragung ist, dass Stress und Belastung mit bestimmten Arbeits- und Organisationsbedingungen zusammenhängen. Von daher muss es um den Abbau eben dieser beeinträchtigender Belastungen und Stressfaktoren gehen. Zugleich geht es um die Bereitstellung - den Herausforderungen entsprechender - personaler, organisationaler und räumlicher Ressourcen, um effektiv delegieren und den Anteil lästiger Routineaufgaben reduzieren zu können. Deutlich in den Aussagen wurde: Hausdirektionen werden ihren anspruchsvollen Aufgaben dann gerecht, wenn sie Zeit hierfür haben und nicht an Verwaltung und Bürokratie ersticken.

- Klima: Neben einer guten Arbeitsorganisation werden ein guter Informationsfluss und Autonomie im Tagesgeschäft sowie die Förderung persönlicher Entwicklungschancen und Spielraum für Gehaltserhöhung

\section{Stichwörter: Burn-out, Stress, Depression}

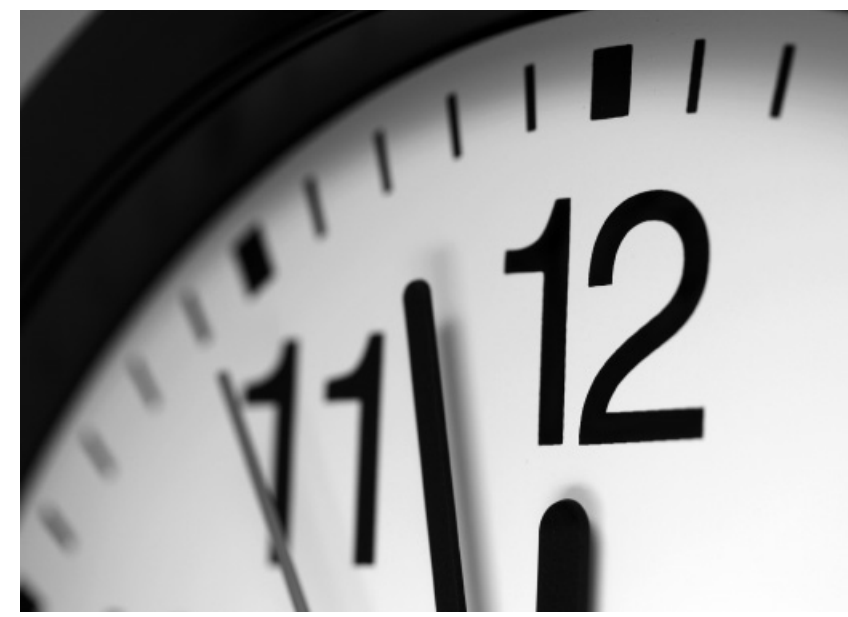

"Burn-out « ist fast eine Modekrankheit unter Angehörigen sozialer Berufsgruppen geworden. Die inflationäre Verwendung des Begriffs erfasst im Kern zwar Wichtiges, verliert aber in der Ausweitung des Alltagsgebrauchs seine Schärfe.

Im Sprachgebrauch wird Burn-out mit Überarbeitung, Überforderung und Erschöpfung gleichgesetzt, was zu Depressivität, Ängsten und Leistungsversagen führt. In der Wissenschaft aber besteht weder Konsens darüber, wie Burn-out entsteht, noch ob es als sinnvolle Bezeichnung eines Krankheitsbildes taugt. Medizinisch-wissenschaftlich ist Burn-out keine klar definierte Krankheit, sondern eher eine Selbstzuschreibung. Durch die unklare Begriffsfassung sind Studien dazu immer kritisch zu betrachten. DieWeltgesundheitsorganisation führt Burn-out erst am Schluss ihres Diagnosesystems ICD (Internationale Klassifikation der
Krankheiten) auf. Dort geht es nicht um Diagnosen, sondern "Faktoren, die den Gesundheitszustand beeinflussen" und jemanden dazu bringen, das Gesundheitssystem in Anspruch zu nehmen. Burn-out fällt unter die Kodierung ICD-10, in der es um Faktoren geht, die "Probleme mit Bezug auf Schwierigkeiten bei der Lebensbewältigung « bereiten. Demnach ist Burn-out zwar ein Problem, aber keine Krankheit.

Inzwischen herrscht die Ansicht vor, dass sowohl organisatorische als auch individuelle psychische Voraussetzungen an der Entstehung von Burn-out beteiligt sind. Uneinigkeit besteht darüber, wie die Dynamik zwischen den Faktoren aussieht und wie sie zu gewichten sind. Den äußeren Faktoren wie hohe Arbeitsbelastung, Zeitdruck, schlechte Arbeitsbedingungen (die subjektiv unterschiedlich wahrgenommen werden) stehen betriebliche Probleme gegenüber: Mangel an Anerkennung, an Autonomie, Handlungs- und Entfaltungsspielraum, an tragfähigen Beziehungen zu Kollegen etc.

Damit dies letztlich zu Burn-out führt, müssen in der Regel noch persönliche Faktoren hinzukommen, wie hohe Ansprüche an sich selbst, unrealistische Situationswahrnehmung, ausgeprägte Wünsche nach Anerkennung, unbewältigter Stress oder ein labiles Selbstwertgefühl. Bei der Entstehung von Burn-out wirken Einflüsse aus all diesen Bereichen zusammen.

Die Prävention von Burn-out muss von daher aufverschiedenen Ebenen ansetzen: an den Rahmenbedingungen der Organisation, im unmittelbaren Arbeitsumfeld, aber auch an den Einstellungen und Verhaltensweisen jedes Einzelnen.

Thomas Mäule 
und Prämien (auch für spontane, um »Sackgassengefühle « zu vermeiden) mit positiven Emotionen assoziiert.

- Vorgesetzte: Auf die Frage nach Unterstützung wurde immer wieder die Rolle des Vorgesetzten angesprochen. Klar formulierte und deutlich ausgesprochene Anerkennung und Wertschätzung dienen der Gesunderhaltung; damit verbunden der Wunsch nach Würdigung und Akzeptanz der geleisteten Arbeit. Rückmeldung durch Vorgesetzte und Rückendeckung sind zentrale Momente eines burn-out-präventiven Führungsstils. Gegebenenfalls sind Fortbildungen für Vorgesetzte angezeigt: zur Steigerung der emotionalen und anderer persönlichen Kompetenzen. $\mathrm{Zu}$ denken ist an Supervision: um an Lösungsstrategien zu arbeiten, die aus der Vorgesetztenrolle sich ergeben oder um Arbeitsklimaprobleme zu thematisieren.

- Arbeitszeitgestaltung: Angesichts von Zeitdruck, Zeitnot, Zeitmangel sind Möglichkeiten ausfindig zu machen, wie durch kreative Problemlösungen exzessive Überstunden, Entgrenzung und Burn-out-Gefährdung verringert werden können. Zeitmanagement (um sogenannte »Antreiber « zu entschärfen) kann ein Ansatzpunkt sein. Das Einhalten von Arbeitszeiten als Selbstschutz ein anderer: Arbeit nicht nach Hause nehmen, Urlaubszeiten einhalten. Ein weiterer: großzügige Arbeitszeitregelungen, Freistellen für wichtige Ereignisse, Sabbat-Monate mit Kopplung an das Einkommen etc.

- Kollegialität und Zusammenarbeit: Als immens wichtiger Einflussfaktor werden die Kollegenschaft und ein gutes Team genannt. Teambesprechungen, Teamtraining, das Erarbeiten gemeinsamer Ziele, gemeinsam feiern, Austausch von Teams etc. können wichtige Momente sein.

- Beratung und Unterstützung: Gerade für neue Führungskräfte (knapp 40\% der Hausdirektionen in der Evangelischen Heimstiftung sind kürzer als fünf Jahren in dieser Führungsposition tätig) können individuelle Beratung und Unterstützung hilfreich sein. Dies kann in kollegialem Rahmen erfolgen, ebenso in Form des Coachings. Ziel des Mentorings muss sein, Leitungskräfte mit den Realitäten der Hausdirektoren-Rolle vertraut zu machen und sie Schritt für Schritt an die neuen Arbeitsanforderungen heranzuführen.

\section{Was wer tun kann: die Organisation}

Auf Organisations- und Trägerebene sind individuelle Erwartungen und institutionelle Strukturen in Einklang zu bringen. Hier sind

- Rahmenbedingungen (»Eindämmen der Bürokratie «, »Arbeitszeitmodelle «) zu schaffen und

- Strukturen und Verantwortlichkeiten (»Schnittstellen « und Zuständigkeiten, »Fachkräfteschlüssel «) zu klären

- Trainingsprogramme zu initiieren und dafür Führungskräfte freizustellen. Als Burn-out-Prävention haben sich Trainings in Kommunikation, Konfliktbewältigung, Team- und Zeitmanagement bewährt.
Die uneingeschränkte Verantwortung für viele verschiedene Aufgaben geht mit schlecht planbaren Arbeitszeiten und Überstunden einher.

Die Zusammenarbeit mit den Vorgesetzten wird einerseits von zahlreichen Hausdirektionen als große Stütze, andererseits von vielen als belastend wahrgenommen.

Zuverlässige Mitarbeitende und ein gutes Betriebsklima sind wesentliche Ressourcen, die durch Knappheit und zunehmende Belastung der Mitarbeiterden als gefährdet betrachtet werden.

Die Hauptverwaltung wird stark mit Bürokratie und Kontrollzwängen in Verbindung gebracht, während die fachliche Unterstützung sowie die Ausbildungs- und Betreuungsangebote hoch geschätzt werden.
Klärung des Aufgaben- und Anforderungsprofils der Hausdirektion:Was sind Kernaufgaben? Was an Voraussetzungen ist erforderlich? Wie lässt sich mit verschiedenen Erwartungen umgehen? Was wäre in den Augen der Hausdirektionen ein realistisches und attraktives Zukunftsszenario?

Klärung der Vorgesetztenrolle und Erarbeitung eines Führungskonzeptes: Was zeichnet gute und erfolgreiche Führung aus? Was heißt persönliche Begleitung, was Rückendeckung? Wie kommunizieren Führungskräfte richtig? Wie können sie kreatives Potenzial der Hausdirektionen aktivieren? Wie können Trainings helfen? Welche Angebote sind hierfür bereitzustellen?

Klärung der Rahmenbedingungen für ein gutes Betriebsklima: Wie viel und welches Personal benötigt die jeweilige Einrichtung? Wie müssen Teams zusammengesetzt sein? Was macht sie erfolgreich? Was brauchen sie an Unterstützung? Wie lassen sich unangenehme Aufgaben auf alle verteilen? Wie lassen sich Vertretungsmöglichkeiten zur Entlastung unersetzlicher Spezialisten schaffen?Wie können Vertretungen rasch verfügbar sein? "Stress und Burn-out" könnten in Mitarbeiterbefragungen thematisiert und in der Mitarbeiterschaft offen und öffentlich diskutiert werden.

Klärung der Rolle der Hauptverwaltung als Schnittstelle zwischen Träger und Einrichtung: Was wird von Fachabteilungen für das Tagesgeschäft vor Ort konkret benötigt? Was kann verschlankt, was sollte weiter ausgebaut werden? Wie kann Bürokratie eingedämmt werden? Was sind Hilfen bei Stressbewältigung, Möglichkeiten der Supervision, Angebote an Fortbildungen und Schulungen?

Bei der Betrachtung der Befragungsergebnisse in der Evangelischen Heimstiftung zu Dauerbelastungen von Führungskräften lassen sich Handlungsfelder identifizieren, die für die Hausdirektionen besonders relevant sind. Zu den vier meist genannten Problemfeldern wurden Anregungen und Ideen für die Weiterarbeit formuliert. 
Der Bedarf an begleiteter Reflexion spricht dafür,

- Supervision und Coaching für alle Einrichtungsleitenden und deren Vorgesetzte zu ermöglichen.

Empfohlen wird schließlich,

- das Thema »Burn-out « trägerweit zu thematisieren. Der Anspruch an Hausdirektionen, gesundheitsfördernd $\mathrm{zu}$ führen, darf nicht im stressigen Arbeitsalltag untergehen.

\section{Fazit}

Keiner der beschriebenen Ansätze stellt ein Patentrezept für Burn-out-Prävention dar. Jede Maßnahme hat jedoch den Vorteil, zugleich die Gesundheit der Füh-

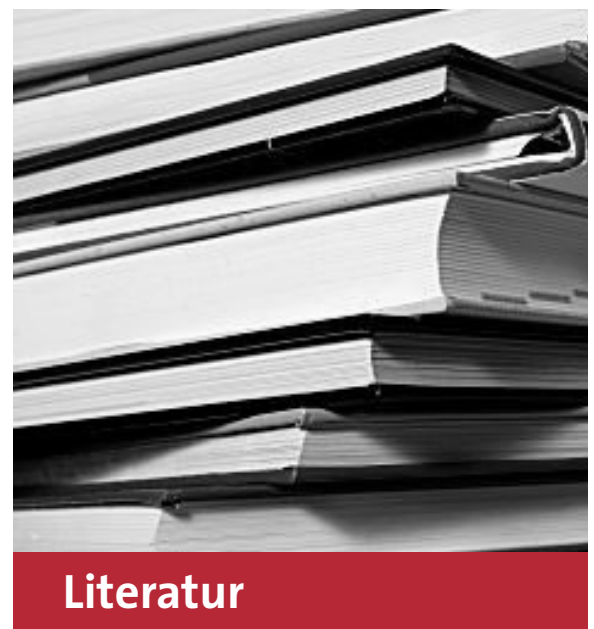

Bergner, T.M.H.: Burnout bei Ärzten. Arztsein zwischen Lebensaufgabe und Lebens-Aufgabe. 2., aktualisierte Aufl., Stuttgart $2010 a$.

Bergner, T.M.H.: Burnout-Prävention. Sich selbst helfen - das 12-Stufen-Programm, 2., aktualisierte Aufl., Stuttgart 2010b.

Burisch, M.: Das Burnout-Syndrom. Theorie der inneren Erschöpfung. 4., aktualisierte Aufl., Heidelberg 2010.

BZgA (Bundeszentrale für gesundheitliche Aufklärung): Was erhält Menschen gesund? Antonovskys Modell der Salutogenese.

Diskussionsstand und Stellenwert. Köln 2001.

Gerstner, A., Gesundheitsfördernd führen. In: H. Schambortski (Hg.): Mitarbeitergesundheit und Arbeitsschutz. Gesundheitsförderung als Führungsaufgabe, München 2008, S. 5-18. Poulsen, I.: Burnoutprävention im Berufsfeld Soziale Arbeit. Perspektiven zur Selbstfürsorge von Fachkräften, Wiesbaden 2009.

Rösing, I.: Ist die Burnout-Forschung ausgebrannt? Analyse und Kritik der internationalen Burnout-Forschung, Heidelberg 2003. rungskräfte zu steigern und die Mitarbeiter- und Kundenzufriedenheit zu fördern. Persönlichkeit und Stil von Hausdirektionen prägen die Einrichtung und beeinflussen das Verhalten der Mitarbeitenden. Burn-out-Prävention ist von daher Schlüsselfaktor für eine gesunde Organisationskultur.

\title{
Stiftungswesen in Deutschland
}

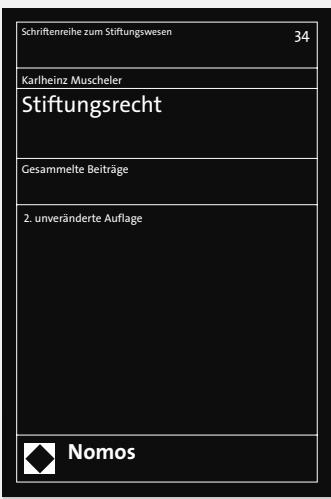

\author{
Stiftungsrecht \\ Gesammelte Beiträge \\ Von Prof. Dr. Karlheinz Muscheler \\ 2. unveränderte Auflage 2011, 393 S., brosch., 98,- $€$ \\ ISBN 978-3-8329-6613-3 \\ (Schriftenreihe zum Stiftungswesen, Bd. 34)
}

Das Stiftungswesen hat in Deutschland in den letzten Jahren einen großen Aufschwung erlebt. Das Buch behandelt alle wichtigen Probleme des Stiftungsrechts in grundlegender Weise. Theoretiker und Praktiker des Stiftungsrechts sind gleichermaßen angesprochen.

Weitere Informationen: www.nomos-shop.de/13637

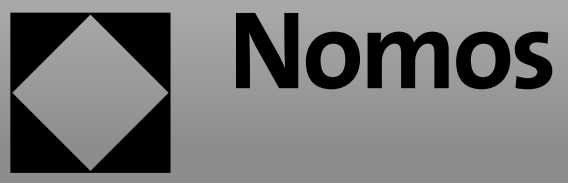

